\title{
O Princípio da Máxima Entropia e a Moderna Teoria das Carteiras
}

\author{
Ailton Cassettari*
}

\section{Resumo}

Neste trabalho, desenvolveu-se uma metodologia de alocação de capital baseada no 'Princípio de Máxima Entropia'. A Entropia de Shannon é utilizada como medida de risco financeiro e comentadas suas vantagens e limitações em relação a outras medidas, no âmbito da Moderna Teoria das Carteiras. Particularmente, a metodologia é testada fazendo-se uma comparação sistemática com duas abordagens: 1) a clássica médiavariância (Markowitz) e 2) a média-VaR (as alocações de capital baseadas no conceito de 'Value at Risk', aqui usando-se o VaR - Hiperbólico). Tais confrontações mostraram-se plenamente coerentes e, em princípio, atestam a plausibilidade e efetividade do método desenvolvido.

\begin{abstract}
In this work, a capital allocation methodology based on the 'Principle of Maximum Entropy' was developed. The Shannons entropy is used as a measure of financial risk. In addition, the advantages and limitations of this measure, concerning the Modern PortfolioTheory, are also discuted. Particularly, the methodology is tested making a systematic comparison to: 1) the mean-variance (Markowitz) approach and 2) the mean-VaR approach (capital allocations based on the 'Value at Risk' concept). In principle, such confrontations shows the plausibility and effectiveness of the developed method.
\end{abstract}

Palavras chave: alocação de capital, otimização de carteiras, medidas de risco.

JEL Code: C61, G11, G14.

\footnotetext{
*Banco Sudameris-Brasil S/A, Av. Paulista, 1000, 4 ${ }^{\underline{Q}}$ and., 01310-100 São Paulo, ailton.cassettarijr@sudameris.com.br
}

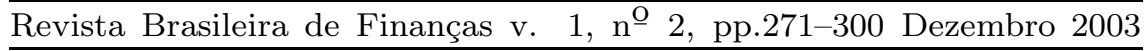




\section{Introdução.}

Uma das mais importantes questões em Finanças diz respeito à construção e gerenciamento de carteiras de risco. $\mathrm{Cu}-$ riosamente, desde o trabalho pioneiro de Markowitz, nos anos cinqüenta, pode-se dizer que a teoria da otimização de carteiras não sofreu radicais alterações. A despeito da extraordinária evolução dos computadores e das técnicas de cálculo, a abordagem média-variância parece não perder sua importância, seja por sua simplicidade de formulação, seja por sua eficácia no gerenciamento de ativos.

De acordo com a teoria de Markowitz (1959), um portifólio eficiente é dado pela solução do seguinte problema de otimização:

$$
\sup _{\alpha} \alpha^{T} \mu
$$

sujeito a:

$$
\left\{\begin{array}{l}
\alpha^{T} \sum \alpha \leq s \\
\alpha^{T} \frac{1}{1}=1
\end{array}\right.
$$

em que $\mu$ é o vetor de retornos esperados dos $\mathrm{N}$ ativos que compõem a carteira e $\sum$ é a correspondente matriz de correlação. Ou seja, o problema do investidor é maximizar o retorno esperado para uma dada variância $s$, ou, alternativamente, para um dado retorno esperado, minimizar a variância correspondente. Como é óbvio, nessa formulação, a variância corresponde à medida de risco, o que implica que o mundo é gaussiano. Há considerável evidência, entretanto, que os retornos dos ativos não são normais, e portanto, a aproximação gaussiana pode ser ruim em muitas situações.

Recentemente, foram sugeridas algumas alternativas à teoria de Markowitz. As mais simples utilizam a mais corriqueira 
medida de risco, o VaR, como substituição à clássica variância (por exemplo, [2] ). Outras, bem mais complexas, são, em sua maioria, baseadas na noção de 'downside risk'. No segundo caso, um conceito muito utilizado é o de 'lower partial moments' (LPM), definido como:

$$
\operatorname{LPM}_{n}(x)=\int_{-\infty}^{x}(x-r)^{n} d F(r), \quad x \in \mathcal{R}
$$

em que $L P M_{n}$ é o LPM de ordem $n, r$ é o retorno do portifólio e $F$ é a distribuição de probabilidade desses retornos. Vários exemplos e conseqüências dessas medida podem ser encontrados, por exemplo, em Fishburn (1977) e Harlow (1991).

Também recente é a noção de 'medida de risco coerente', introduzida por Artzner, Delbaen, Eber e Heath (1999). Estes autores buscaram fornecer uma definição de risco (de mercado e também outros) e chegaram a um esquema unificado que reúne a análise, construção e implementação de 'medidas' de risco. Como resultado, surge metodologia apta a caracterizar uma grande classe de medidas de risco. No dizer desses autores, a escolha de uma particular medida a ser utilizada em um dado problema deverá, presumivelmente, ser feita à luz de condições econômicas adicionais e das peculiaridades de cada situação.

O objetivo principal do presente trabalho é utilizar o conceito da entropia de Shannon como medida de risco e avaliar seu impacto na otimização estática de portifólios. Em outras palavras, tentaremos avaliar o impacto da substituição do desvio-padrão por essa quantidade, mantendo a abordagem de Markowitz. Mostraremos que essa medida satisfaz aos principais requerimentos da teoria das carteiras e traz algumas vantagens em relação ao desvio-padrão. Serão feitas também confrontações com a própria abordagem média-variância bem como com outra metodologia de alocação de capital, e mostradas a plausibilidade e efetividade da presente abordagem. 


\section{O Princípio da Máxima Entropia.}

A Entropia tem um significado matemático e físico preciso, embora muitas vezes ela seja envolta em um injustificado mistério. A seguir, faremos um breve comentário a respeito desse conceito para mais tarde avaliar sua aplicação à Teoria das Carteiras.

Em Física, a entropia de um sistema é uma medida de sua 'desordem'. A lei física associada a esse conceito é que a entropia de um sistema, desde os mais simples aos mais complexos, deve sempre aumentar. Essa lei (mais conhecida como 'Princípio da Máxima Entropia') reflete a tendência natural das coisas de se aproximar do estado caótico, a menos que algo atue sobre elas para evitar isso. Erwin Schrödinger ${ }^{1}$ escreve, a propósito, que "todo processo, evento, ocorrência chame-se-lhe como se quiser - numa palavra, tudo o que acontece na natureza significa um aumento da entropia da parte do mundo onde acontece".

O físico austríaco Ludwig Boltzmann definiu a entropia de um sistema através da seguinte expressão:

$$
S=k \cdot \ln \Omega
$$

em que $k$ é uma constante (positiva) de ajuste dimensional e $\Omega$ é o número de estados do sistema. A 'desordem' (que denotaremos por D) a que nos referimos anteriormente está diretamente relacionada ao número de estados, o que, aliás, é intuitivo. Então,

$$
S=k \cdot \ln D
$$

\footnotetext{
${ }^{1}$ Erwin Schrödinger- 'O que é vida?' (Ed. da Unesp, 1997).
} 
Se $D$ é uma medida de desordem seu recíproco, $1 / D$, é uma medida de ordem. Portanto, se $S$ mede a desordem, $-S$ (uma entropia negativa) mede a ordem do sistema.

Uma das mais importantes variantes da equação (4) é a 'entropia de Shannon', definida como [6]:

$$
H(X)=-k \cdot \sum_{i=1}^{n} p_{i} \ln p_{i}
$$

em que $H(X)$ é a entropia da variável aleatória $X$, denota a probabilidade de que $X$ esteja no estado ' $i$ ' , $n$ é o número de estados possíveis e $k$ é uma constante para ajuste dimensional.

A expressão (5) desempenha um papel central na Teoria da Informação, como uma medida de informação, escolha e incerteza. Nessa teoria, $H$ mede, em algum sentido, quanta informação é produzida num processo ou, mais precisamente, em que taxa produz-se informação. As principais propriedades da Entropia de Shannon são apresentadas no Apêndice A.

\section{A Entropia de Shannon e a Medida do Risco Finan- ceiro.}

Como vimos, a entropia de Shannon desempenha, na Teoria da Informação, entre outros, o papel de uma medida de incerteza. Nosso objetivo, nessa seção, é estendê-la como uma medida de risco financeiro.

Inicialmente, façamos a seguinte

\section{Definição 1:}

$O$ risco $(X)$ de um ativo financeiro é uma variável aleatória limitada que representa o retorno esperado desse ativo, num dado horizonte de tempo. A medida desse risco é: 


$$
H(X)=-\frac{K}{\ln (\sqrt{2 \pi \cdot e})} \cdot \sum_{i=1}^{n} p_{i} \ln p_{i}
$$

em que a constante $K$ é o capital em exposição ao risco, $p_{i}$ é a probabilidade associada a $X$, num dado ponto do tempo e $e$ é a base dos logaritmos neperianos.

No que segue, demonstraremos que $H$ satisfaz às propriedades desejáveis como medida de risco a ser utilizada na otimização de carteiras² .

Proposição 1.1: $-H$ é sub-aditiva

Seja um conjunto de variáveis aleatórias limitadas $X, Y, Z, \ldots$ e $H(X, Y, Z, \ldots)$ a entropia conjunta. Da propriedade $\mathbf{P 3}$ da entropia de Shannon, segue que:

$$
H(X, Y, Z, \ldots) \leq H(X)+H(Y)+H(Z)+\ldots
$$

Proposição 1.2: $H$ satisfaz à homogeneidade positiva Seja $\lambda>0$ e um conjunto de $\lambda$ variáveis aleatórias limitadas $X, Y, Z, \ldots$ Da propriedade P3, a entropia conjunta é (sem perda de generalidade, a constante pode ser feita unitária):

\footnotetext{
${ }^{2}$ Em pesquisa recente, Artzner, Delbaen, Eber e Heath [5] estabeleceram uma série de propriedades que uma medida de risco deveria satisfazer. Em relação à teoria das carteiras, as mais imprescindíveis são a sub-aditividade e a homogeneidade positiva. A primeira assegura o efeito da 'diversificação', segundo o qual o risco da carteira é sempre no máximo igual à soma dos riscos dos ativos individuais que a compõem. A segunda reflete precisamente a situação em que o efeito da diversificação (embora exista) é completamente anulado (conferir Apêndice B do presente trabalho). Pode-se facilmente verificar que o desvio-padrão também satisfaz a essas duas propriedades, diferentemente do VaR.
} 
$H(X, Y, Z, \ldots)=\sum p(X, Y, Z, \ldots) \cdot \ln [p(X, Y, Z, \ldots)]$

Se as variáveis são independentes, temos:

$$
p(X, Y, Z, \ldots)=p(X) \cdot p(Y) \cdot p(Z) \ldots
$$

Se $p(X)=p(Y)=p(Z)=\ldots$, então,

$$
H(X, Y, Z, \ldots)=\sum p(X, Y, Z, \ldots) \cdot \ln \left[(p(X))^{\lambda}\right]
$$

o que pode ser escrito:

$$
H(X, Y, Z, \ldots)=\lambda . \sum p(X, Y, Z, \ldots) \cdot \ln [p(X)]
$$

Da equação (A2),

$$
H(X)=\sum p(X, Y, Z, \ldots) \cdot \ln [p(X)]
$$

Portanto, (10) fica:

$$
H(X, Y, Z, \ldots)=\lambda . H(X)
$$

Além dessas importantes propriedades no âmbito da teoria das carteiras, deve-se notar a seguinte:

Proposição 1.3: se $X$ é uma variável aleatória gaussiana, com média 0 e desvio-padrão $\sigma$, então,

$$
H(X)=K \cdot \sigma
$$


O Princípio da Máxima Entropia e a Moderna Teoria das Carteiras

Se $p(X)$ é contínua, a equação (6) é escrita:

$$
H(X)=-\frac{K}{\ln (\sqrt{2 \pi \cdot e})} \cdot \int p(X) \cdot \ln p(X) d x
$$

Se $p(X)$ é gaussiano,

$$
p(X)=\frac{1}{\sqrt{2 \pi \sigma}} e^{-\left(X^{2} / 2 \sigma^{2}\right)}
$$

e

$$
-\ln p(X)=\ln \sqrt{2 \pi} \sigma+\frac{X^{2}}{2 \sigma^{2}}
$$

Portanto,

$$
\begin{aligned}
H(X) & =\frac{K}{\ln \sqrt{2 \pi e}} \cdot\left\{\int p(X) \ln \sqrt{2 \pi} \sigma \cdot d X+\int p(X) \cdot \frac{X^{2}}{2 \sigma^{2}} d X\right\} \\
& =\frac{K}{\ln \sqrt{2 \pi e}}\left(\ln \sqrt{2 \pi} \sigma+\frac{\sigma^{2}}{2 \sigma^{2}}\right) \\
& =\frac{K}{\ln \sqrt{2 \pi e}} \cdot(\ln \sqrt{2 \pi} \sigma+\ln \sqrt{e}) \\
& =\frac{K}{\ln \sqrt{2 \pi e}} \cdot \ln \sqrt{2 \pi e} \cdot \sigma
\end{aligned}
$$

o que implica que

$$
H(X)=K . \sigma
$$




\section{O Princípio da Máxima Entropia e a Teoria das Carteiras.}

Seja ПI uma carteira composta por $n$ ativos financeiros quaisquer. Da propriedade (P3), podemos escrever, para П:

$$
\begin{aligned}
& \left(k_{1}+k_{2}+\ldots+k_{n}\right) \cdot \triangle\left(X_{1}, X_{2}, \ldots, X_{n}\right) \leq k_{1} \cdot \triangle\left(X_{1}\right) \\
& \quad+k_{2} \cdot \triangle\left(X_{2}\right)+\ldots+k_{n} \cdot \triangle\left(X_{n}\right)+
\end{aligned}
$$

em que

$$
\triangle\left(X_{j}\right)=-\left(\frac{1}{\ln (\sqrt{2 \pi e})}\right) \cdot \sum_{i} p_{i}\left(X_{1}, X_{2}, \ldots, X_{n}\right) \cdot \ln p_{i}\left(X_{j}\right)
$$

e $k_{i}$ é o capital investido no ativo $X_{i}$. A equação (15) pode ser escrita como:

$$
\begin{aligned}
& \triangle\left(X_{1}, X_{2}, \ldots, X_{n}\right) \leq \frac{k_{1}}{\left(k_{1}+k_{2}+\ldots+k_{n}\right)} \cdot \triangle\left(X_{1}\right)+ \\
& \frac{k_{2}}{\left(k_{1}+k_{2}+\ldots+k_{n}\right)} \cdot \Delta\left(X_{2}\right)+ \\
& \frac{k_{3}}{\left(k_{1}+k_{2}+\ldots+k_{n}\right)} \cdot \triangle\left(X_{3}\right)+\ldots+ \\
& \frac{k_{3}}{\left(k_{1}+k_{2}+\ldots+k_{n}\right)} \cdot \triangle\left(X_{n}\right)
\end{aligned}
$$

Fazendo as seguintes abreviações: 


$$
\begin{aligned}
& \omega_{1}=\frac{k_{1}}{\left(k_{1}+k_{2}+\ldots+k_{n}\right)} \\
& \omega_{2}=\frac{k_{2}}{\left(k_{1}+k_{2}+\ldots+k_{n}\right)} \\
& \cdot \\
& \omega_{n}=\frac{k_{n}}{\left(k_{1}+k_{2}+\ldots+k_{n}\right)}
\end{aligned}
$$

o que implica que

$$
\omega_{1}+\omega_{2}+\ldots+\omega_{n}=1
$$

A seguir faremos a seguinte

Definição 2: $O$ risco associado à carteira $\Pi$ é dado por:

$$
R_{\Pi}=\sqrt{\sum_{i=1}^{n} \sum_{j=1}^{n} \omega_{i} \omega_{j} \rho_{i j} \triangle\left(X_{i}\right) \triangle\left(X_{j}\right)}
$$

em que $\triangle\left(X_{i}\right)$ é dado por (16) e $\rho$ é o coeficiente de correlação entre os ativos individuais (dois a dois).

Com a definição 2, o problema (1) pode ser formulado como segue:

$$
\inf _{\{\omega\}}\left\{R_{\Pi}\right\}
$$

sujeito a:

$$
\left\{\begin{array}{l}
\sum_{i=1}^{n} \omega_{i} \cdot \mu_{i}=r_{A} \\
\sum_{i=1}^{n} \omega_{i}=1
\end{array}\right.
$$


em que $\mu_{i}$ é o retorno esperado do ativo ' $i$ ' e $r_{A}$ é o retorno requisitado da carteira ('retorno alvo'), ambos num dado período de tempo.

Dessa definição segue a seguinte

\section{Proposição 2.1(Recuperação da solução de Markowitz):} Se $X_{1}, X_{2}, \ldots, X_{n}$ são variáveis aleatórias gaussianas, com média 0 e desvio-padrão $\sigma_{1}, \sigma_{2}, \ldots, \sigma_{n}$, então, a solução do problema (21) é idêntica à solução do problema (1).

A prova segue diretamente da proposição 1.3.

\section{Exemplos de Aplicação.}

a) A Otimização de uma Carteira de Ativos de Renda Variável.

Nesta seção, a título de exemplo, vamos otimizar uma carteira fictícia composta de 4 ativos A,B,C e D (vide Apêndice C), com o auxílio da metodologia supra desenvolvida.

Inicialmente, para cada um dos ativos, vamos avaliar a expressão (16). Para tanto, serão utilizados dados históricos relativos a períodos recentes do mercado acionário da BOVESPA (cf. Ap. C). De posse desses dados, foram calculados os retornos diários (logaritmos) - denotados por $X$ - inicialmente de cada um dos ativos que compõem a carteira. Esses retornos são então indexados com ' $i$ '. A seguir são construídos os histogramas normalizados desses retornos, obtendo-se assim $p_{i}\left(X_{j}\right), j=$ $A, \ldots, D$. A probabilidade conjunta $p_{i}\left(X_{A}, X_{B}, X_{C}, X_{D}\right)$ é obtida de maneira análoga. As figuras mostram os resultados ${ }^{3}$.

\footnotetext{
${ }^{3}$ Uma questão importante diz respeito à determinação do número de estados. Uma análise aprofundada deste tópico foge ao escopo do presente trabalho. Para uma breve discussão a esse respeito, vide Apêndice D.
} 
Figura I - Histograma de retornos diários do ativo A

Figura II - Histograma de retornos diários do ativo B 
Figura III - Histograma de retornos diários do ativo C

Figura IV - Histograma de retornos diários do ativo D 
Figura V - Histograma de retornos diários da carteira

A Tabela I resume alguns resultados. 
Tabela I

\begin{tabular}{|c|c|c|c|c|c|}
\hline & $\mathrm{A}$ & B & $\mathrm{C}$ & $\mathrm{D}$ & $\begin{array}{l}\text { Portfolio } \\
\text { dos Ativos }\end{array}$ \\
\hline$n$ & $p_{i}$ & $p_{i}$ & $p_{i}$ & $p_{i}$ & $p_{\text {Total }_{i}}$ \\
\hline 1 & 0.001149 & 0.001149 & 0.001149 & 0.001149 & 0.001149 \\
\hline 2 & 0.001149 & - & - & - & - \\
\hline 3 & 0.002299 & - & - & 0.001149 & - \\
\hline 4 & 0.001149 & - & - & - & - \\
\hline 5 & 0.003448 & 0.002299 & 0.001149 & 0.001149 & - \\
\hline 6 & 0.004598 & 0.001149 & - & - & - \\
\hline 7 & 0.008046 & 0.002299 & - & - & 0.001149 \\
\hline 8 & 0.008046 & 0.004598 & 0.002299 & - & 0.004598 \\
\hline 9 & 0.02069 & 0.01954 & 0.001149 & 0.005747 & 0.004598 \\
\hline 10 & 0.037931 & 0.031034 & 0.004598 & 0.010345 & 0.009195 \\
\hline 11 & 0.062069 & 0.162069 & 0.006897 & 0.005747 & 0.006897 \\
\hline 12 & 0.117241 & 0.485057 & 0.013793 & 0.01954 & 0.018391 \\
\hline 13 & 0.181609 & 0.194253 & 0.027586 & 0.025287 & 0.056322 \\
\hline 14 & 0.204598 & 0.058621 & 0.075862 & 0.058621 & 0.122988 \\
\hline 15 & 0.144828 & 0.018391 & 0.136781 & 0.087356 & 0.228735 \\
\hline 16 & 0.072414 & 0.009195 & 0.264367 & 0.151724 & 0.270115 \\
\hline 17 & 0.043678 & 0.004598 & 0.208046 & 0.305747 & 0.155172 \\
\hline 18 & 0.02069 & 0.001149 & 0.147126 & 0.122988 & 0.058621 \\
\hline 19 & 0.028736 & 0.001149 & 0.058621 & 0.074713 & 0.033333 \\
\hline 20 & 0.012644 & - & 0.025287 & 0.051724 & 0.018391 \\
\hline 21 & 0.009195 & - & 0.013793 & 0.027586 & 0.003448 \\
\hline 22 & 0.004598 & - & 0.006897 & 0.01954 & 0.002299 \\
\hline 23 & 0.002299 & 0.001149 & - & 0.012644 & - \\
\hline 24 & 0.001149 & - & - & 0.004598 & 0.002299 \\
\hline 25 & - & - & 0.002299 & 0.008046 & - \\
\hline 26 & - & - & - & 0.001149 & 0.001149 \\
\hline 27 & 0.002299 & 0.001149 & - & 0.001149 & - \\
\hline 28 & 0.001149 & - & - & - & - \\
\hline 29 & 0.001149 & - & - & 0.001149 & - \\
\hline 30 & 0.001149 & 0.001149 & 0.002299 & 0.001149 & 0.001149 \\
\hline Pesos & $1 / 4$ & $1 / 4$ & $1 / 4$ & $1 / 4$ & 1 \\
\hline$H=-1 . \sum p_{\text {Total }} \ln p$ & 2,547303 & 4,193587 & 2,112272 & 2,30147 & 2.03205 \\
\hline Entropia $\times$ peso & 0,6368 & 1,0498 & 0,5280 & 0,5753 & 2.03205 \\
\hline
\end{tabular}


De posse dos dados da tabela anterior podemos passar agora à solução do problema dado por (21). As figuras mostram a composição da carteira obtida pela metodologia desenvolvida, juntamente com outros métodos de alocação ${ }^{4}$, para facilitar a comparação.

Figura VI - Composição para um Retorno alvo de $5 \%$ sobre $r$

Figura VII - Composição para um Retorno alvo de $10 \%$ sobre $r$

\footnotetext{
${ }^{4}$ As outras metodologias às quais nos referimos nas figuras (Markowitz e HIP-95/99\%) acham-se descritas respectivamente nas referncias [1] e [7].
} 
Figura VIII - Composição para um Retorno alvo de $15 \%$ sobre $r$ Figura IX - Composição para um Retorno alvo de $20 \%$ sobre $r$ 
Figura X - Composição para um Retorno alvo de 25\% sobre $r$

As figuras anteriores mostram uma forte semelhana entre as diversas metodologias, como se esperaria. A principal confrontação deve ser feita em relação à teoria de Markowitz, pois releva a distância com a aproximação gaussiana. Tais detalhes serão discutidos mais tarde.

b) O Cálculo da Fronteira Eficiente da Carteira Utilizando a Entropia como medida de risco.

Para o cálculo da fronteira eficiente dos ativos da carteira utilizando a metodologia desenvolvida, dado um conjunto de retornos-alvos fixados, minimiza-se a quantidade $(20)^{5}$. A fronteira eficiente é mostrada na Figura XI. A Tabela II mostra a mesma fronteira obtida com o auxílio de outras metodologias (as unidades são arbitrárias).

\footnotetext{
${ }^{5}$ As minimizações são feitas usando-se o 'Solver' do Microsoft Excel.
} 
Tabela II - Análise risco-retorno

\begin{tabular}{lllll}
\hline \hline \% sobre $r$ & MARKOWITZ ENTROPIA & HIPERB.95\% & HIPERB.99\% \\
\hline 5 & 0,028640901 & 1,423026266 & 0,079319806 & 0,053223529 \\
10 & 0,024544657 & 1,253778216 & 0,067013468 & 0,044275413 \\
15 & 0,023911227 & 1,202394695 & 0,062529533 & 0,040233001 \\
20 & 0,026985572 & 1,283114562 & 0,067446673 & 0,04251928 \\
25 & 0,03273932 & 1,474398083 & 0,080050794 & 0,050278193 \\
30 & 0,040033395 & 1,740158965 & 0,097402128 & 0,061471267 \\
35 & 0,048173125 & 2,051656296 & 0,117414595 & 0,074567751 \\
40 & 0,056796082 & 2,391081467 & 0,138943041 & 0,088728772 \\
45 & 0,065712309 & 2,748105618 & 0,161381902 & 0,103518371 \\
50 & 0,074817029 & 3,11668656 & 0,184399122 & 0,118701828 \\
\hline
\end{tabular}

Para facilitar a comparação, vamos adotar o seguinte procedimento. Para cada uma das metodologias calcula-se um 'risco normalizado', que definimos como:

$$
\text { Risco Normalizado } \equiv \frac{\text { Risco }}{\left(\text { Risco }_{\max }-\text { Risco }_{\min }\right)}
$$

em que Riscomax $_{\max }$ e isco $_{\text {min }}$ são, respectivamente, o maior e o menor valor do risco, em cada metodologia (Tabela II). Esse procedimento leva aos resultados da Tabela III. 
O Princípio da Máxima Entropia e a Moderna Teoria das Carteiras

Tabela III - Análise risco-retorno (normalizado)

\begin{tabular}{|c|c|c|c|c|}
\hline$\%$ sobre $r$ & MARKOWITZ & ENTROPIA & HIPERB.95\% & HIPERB.99\% \\
\hline 5 & 0,562625475 & 0,743369541 & 0,650858075 & 0,678276082 \\
\hline 10 & 0,482158332 & 0,654956665 & 0,549878513 & 0,564242069 \\
\hline 15 & 0,469715168 & 0,628114614 & 0,513085615 & 0,512725911 \\
\hline 20 & 0,530107981 & 0,670281573 & 0,553433165 & 0,541862051 \\
\hline 25 & 0,643135326 & 0,770205479 & 0,656856191 & 0,640740976 \\
\hline 30 & 0,78642106 & 0,909035345 & 0,799232429 & 0,783384556 \\
\hline 35 & 0,946318948 & 1,071757308 & 0,963444579 & 0,95028502 \\
\hline 40 & 1,115709405 & 1,249068395 & 1,140096076 & 1,130751853 \\
\hline 45 & 1,290860901 & 1,435572949 & 1,324217985 & 1,319229232 \\
\hline 50 & 1,469715168 & 1,628114614 & 1,513085615 & 1,512725911 \\
\hline
\end{tabular}

Figura XI - Fronteira Eficiente da Carteira, utilizando como medida de risco a equação (20)

Com os dados da Tabela III, podemos construir as curvas risco-retorno e confrontá-las entre si. Os resultados são mostrados na Figura XII. 
Figura XII - Confronto entre as metodologias de alocação de capital

Mais uma vez, a figura anterior demonstra um acordo plenamente satisfatório entre as metodologias.

A despeito da figura anterior, é instrutivo fazermos uma comparação um pouco mais quantitativa entre a Entropia e Markowitz. Um dos procedimentos cabíveis para tanto seria uma regressão do tipo:

$$
y=a x+b+\varepsilon
$$

na qual a variável dependente é a diferença entre as medidas (normalizadas) Entropia e desvio-padrão e a variável independente é a própria entropia (normalizada), considerando apenas o parâmetro $b$. Isso permite avaliar se, em média, a medida baseada na entropia é consistentemente superior ao desviopadrão. 
Os resultados são mostrados na Figura XIII.

\begin{tabular}{lccl}
\hline \hline $\begin{array}{l}\text { Dif.Média } \\
\text { entre as }\end{array}$ & $\begin{array}{c}\text { Desvio-padrão } \\
\text { medidas }\end{array}$ & Stat $t$ & Valor-P \\
\hline 0.003297587 & 0.00082288 & 4.007375269 & 0.000174631 \\
\hline
\end{tabular}

Figura XIII - Confronto Entropia $\times$ Markowitz

Em particular, note-se que, de acordo com a estatística $t$ (para uma amostra de 61 dados), de fato a entropia se distancia do desvio-padrão, mas não de forma muito pronunciada. Essa regressão, juntamente com as figuras VI a X, nos permitem concluir que, embora o risco da carteira não seja substancialmente diferente nas duas abordagens, a alocação de capital apresenta diferenças consideráveis.

c) A 'Seleção' de Ativos que Compõem uma Carteira ("Stock Picking"). 
A propriedade $\mathbf{P 4}$, equação (A6), permite uma aplicação interessante. Ela fornece um critério quantitativo para a inclusão de ativos à carteira e, portanto, para a própria construção de uma carteira de investimentos, baseado na efeito da diversificação. Isso pode ser feito como no exemplo que segue. Seja inicialmente uma carteira qualquer, C, e dois ativos disponveis, A e B, dos quais tem-se de escolher um deles de modo a se obter um melhor efeito de diversificação. Pela abordagem de Markowitz, a maneira de se fazer isso é calcular a correlação entre A (ou B) e todos os ativos que compõem a carteira, e então escolher o que forneceu uma menor variância para C.

Como dissemos, um critério possível é, usando a equação (A5), calcular a entropia relativa de cada ativo disponível em relação à carteira. Pelo fato de o risco de cada ativo estar relacionado a toda a carteira, um critério possível consiste em escolher o de menor entropia relativa, ou seja, aquele que 'injeta' mais entropia negativa à carteira.

\section{Conclusões e Comentários.}

Como enfatizado anteriormente, nosso objetivo neste trabalho foi o de desenvolver uma metodologia de alocação de capital, utilizando o conceito de entropia como medida de risco financeiro. Neste contexto, a entropia de Shannon, pelas suas propriedades, se nos apresenta como uma medida, se não 'coerente' (no sentido da referência em Artzner, Delbaen, Eber e Heath (1999)), pelo menos bastante aceitável e sobretudo útil à teoria das carteiras. Cabe aqui ressaltar alguns pontos marcantes da presente abordagem. O primeiro, e mais importante, é o fato de que não se assume de antemão qualquer distribuição de probabilidades, seja contínua ou discreta. Isso, sem dúvida, confere um grau muito grande de realismo à metodologia (basta lembrar que no caso de Markowitz o mundo 
é gaussiano). Não obstante, a solução de Markowitz é recuperada quando se assume a aproximação gaussiana (proposição 2.1). De outra parte, a equação (16) fornece uma ferramenta poderosa para capturar o efeito da diversificação, de suma importância na teoria das carteiras, como vimos. De um ponto de vista qualitativo, parece intuitivo que tal efeito seja conseguido com a 'injeção' de informação à carteira: quanto mais ativos disponíveis, mais informações estão reunidas e, portanto, pelo menos em princípio, o risco deve ser reduzido. Mais precisamente, adicionar informação à carteira é alimentá-la com entropia negativa. Outro aspecto a salientar é a possibilidade de aplicar o método a uma ampla variedade de carteiras, como, por exemplo, as de crédito, para as quais a aproximação gaussiana é ainda mais descabida.

Portanto, de forma geral, pode-se dizer que a metodologia é útil sobremaneira quando se podem inferir as distribuições de probabilidades, individuais e conjunta. No caso dos 'riscos de mercado' isso é razoavelmente simples, como pudemos exemplificar. Em relação a outras carteiras pode ser mais problemático, porém raramente impossível de ser feito.

Submetido em Abril de 2003. Revisado em Outubro de 2003.

\section{Referências}

Artzner, P., Delbaen , F., Eber, J-M \& Heath, D. 1999. "Coherent Measures of Risk". Mathematical Finance, 9, 203228.

Cassettari, A. 2001. "Uma Forma Alternativa para Alocação Ótima de Capital em Carteiras de Risco RAUSP. Revista de Administração, São Paulo, 36(3), julho/setembro.

Fishburn, P. C. 1977. "Mean-risk analysis with risk associated with below-target returns". American Economic Review, 67, 116-126. 
Gaivoronski, A. \& Pflug, G. 2000. Finding Optimal Portifolios with Constraints on Value-at-Risk. Proc. of III Stockhlom Seminar on Risk Management.

Harlow, W. 1991. "Asset Allocation in a Downside-risk Framework". Financial Analysts Journal, 28-40.

Markowitz, H. 1959. Portfolio Selection. John Wiley \& Sons.

Shannon, C. E. 1948. "A Mathematical Theory of Communication". Bell System Technical Journal, 27, 379-423. 


\section{Apêndice A}

\section{Principais Propriedades da Entropia de Shannon.}

No que segue, vamos enunciar de forma bastante sucinta as principais propriedades da entropia de Shannon (equação (5)). Mais detalhes podem ser encontrados em [6].

P1 $H=0$ se e somente se todos os $p_{i}$ são zero, com exceção de um que tem valor unitário. Intuitivamente, essa é a situação de maior certeza. De outra maneira, $H$ é positivo.

P2 Para um dado $n, H$ é máximo e igual a $\ln (n)$ quando todos os $p_{i}$ são iguais (i. é., $1 / n$ ). Contrariamente à situação anterior, esse é o caso de maior incerteza.

P3 Se existem dois eventos, $X$ e $Y$, com $m$ possibilidades para o primeiro e $n$ para o segundo e $p_{i, j}$ é a probabilidade de ocorrência conjunta de ' $i$ ' para o primeiro e ' $j$ ' para o segundo, a entropia do evento conjunto é:

$$
H(X, Y)=-\sum_{i, j} p(i, j) \cdot \ln p(i, j)
$$

com

$$
\begin{aligned}
& H(X)=-\sum_{i, j} p(i, j) \cdot \ln \sum_{j} p(i, j) \\
& H(Y)=-\sum_{i, j} p(i, j) \cdot \ln \sum_{i} p(i, j)
\end{aligned}
$$

Destas definições segue que:

$$
H(X, Y) \leq H(X)+H(Y)
$$


P4 Por definição, a entropia condicional de $Y$ é dada por:

$$
H_{x}(Y)=-\sum_{i, j} p(i, j) \cdot \ln p_{i}(j)
$$

Desta definição resulta,

$$
H(X, Y)=H(X)+H_{x}(Y)
$$

$\mathrm{e}$

$$
H(Y) \geq H_{x}(Y)
$$

\section{Apêndice B}

\section{Medidas Coerentes de Risco.}

$\mathrm{Na}$ referência [5], risco é definido como uma variável aleatória $X$ que representa o ganho líquido de uma Instituição numa certa data futura. Seguindo a mesma referência, denotemos por $L^{\infty}(\Omega, A, P)$ um espao de probabilidades de uma variável aleatória limitada e por $\rho(X)$ a medida de risco, definida nesse espaço.

Em seus artigos, Artzner, Delbaen, Eber e Heath sugeriram um conjunto de propriedades que uma medida de risco deveria satisfazer, assim introduzindo o conceito de medidas coerentes de risco. Por definição, uma medida de risco $\rho: L^{\infty} \rightarrow \mathcal{R}$ é coerente se satisfaz às seguintes propriedades:

1) Sub-aditividade:

Se $X$ e $Y$ são variáveis aleatórias limitadas.

$$
\rho(X+Y) \leq \rho(X)+\rho(Y)
$$


2) Homogeneidade Positiva:

Para todo $\lambda \geq 0$ e para toda variável aleatória limitada $X$,

$$
\rho(\lambda X)=\lambda \cdot \rho(X)
$$

3) Invariância por Translação

Para todo $\alpha \in \mathcal{R}$ e toda variável aleatória limitada $X$,

$$
\rho\left(X+\alpha \cdot r_{f}\right)=\rho(X)-\alpha
$$

em que $r_{f}$ é o preço, numa certa data futura, de um investimento livre de risco cujo preço é 1 hoje.

4) Monotonicidade:

Para toda variável aleatória limitada $X$ e $Y$, tal que $X \leq Y$,

$$
\rho(X) \geq \rho(Y)
$$

As propriedades enunciadas acima devem ser entendidas como condições necessárias para que uma dada medida de risco seja admissível (ou razoável).

\section{Apêndice C}

\section{Dados Históricos e Análises Estatísticas.}

Foram tomados os retornos diários (logaritmos) das ações da Bovespa correspondentes aos seguintes períodos:

ATIVO A: Ação ACES4: 01/07/96 - 27/03/00

ATIVO B: Ação ARCZ6: 01/07/96 - 30/12/99

ATIVO C: Ação CMIG4: 01/07/96 - 30/12/99

ATIVO D: Ação CSNA3: 01/07/96 - 30/12/99

As correlações estimadas, bem como os testes estatísticos pertinentes para os ativos utilizados, constam nos quadros que seguem. 
Matriz de Correlação

\begin{tabular}{lllll}
\hline \hline Ativo & A & B & C & D \\
\hline A & 1 & 0.125803 & 0.405313 & 0.263272 \\
B & 0.125803 & 1 & 0.216858 & 0.144988 \\
C & 0.405313 & 0.216858 & 1 & 0.391812 \\
D & 0.263272 & 0.144988 & 0.391812 & 1 \\
\hline
\end{tabular}

$\begin{array}{lllll} & \mathrm{A} & \mathrm{B} & \mathrm{C} & \mathrm{D} \\ \text { ASSIMETRIA } & 0.562222 & 2.5984784 & -0.03163 & -0.02235 \\ \text { CURTOSE } & 6.928775 & 37.42037 & 11.13652 & 7.268964 \\ \text { JARQUE-BERA } & 645.7197 & 43,926.67 & 2,400.00 & 660.6942 \\ \text { p-valor(doJ-B) } & 6.08 \mathrm{E}-141 & 0.00 \mathrm{E}-00 & 0.00 \mathrm{E}-00 & 3.40 \mathrm{E}-144\end{array}$

\section{Apêndice D}

\section{Determinação do número de estados do sistema.}

Do ponto de vista prático, a aplicação da metodologia desenvolvida depende da determinação do número de estados do sistema, ou, em outras palavras, da distribuição empírica de probabilidade dos retornos. Sem pretensão de ir bastante fundo no assunto, que foge ao escopo deste trabalho, limitar-nos-emos a indicar uma metodologia plausível para tanto.

Como vimos, no exemplo que apresentamos, em que pese a possível existência de algum critério econômico, é lícito admitir que os estados correspondem às abscissas do histograma de retornos dos ativos considerados. Como é bem conhecido, um histograma dá idéia de como é a verdadeira densidade de freqüências da população da qual os dados foram selecionados. Se se adota esse critério, o problema que se apresenta é qual o "melhor histograma" que pode ser levantado empiricamente.

Recentemente, D. Freedman \& P. Diaconis ${ }^{6}$ mostraram que o 'número de estados' obtidos dessa maneira é dado por:

\footnotetext{
6 "On the maximum deviation between histogram and underlying density", Zeitscrift für Wahrscheinlichkeits Theorie und Verwandte Gebiete, 58, p. 139-167. (1981).
} 


$$
\text { no.de estados }=\frac{\left(x_{\max }-x_{\min }\right)}{1,394 . \sigma_{p} \cdot\left(\frac{\log n}{n}\right)^{1 / 3}}
$$

em que $x_{\max }$ e $x_{\min }$ são, respectivamente, os valores máximo e mínimo dos dados, $\sigma_{p}$ é um estimador robusto do desvio-padrão populacional e $n$ é o tamanho da amostra.

Tomando como exemplo o ativo A (ACES4), temos:

No. de estados $=(0,2323-(-01735)) / 1.394 \times 0,04117$

$$
\times(\ln (928) / 928)) \wedge(1 / 3) \cong 37
$$

Desse modo, o número de estados utilizados é bastante próximo do estabelecido por Freedman e Diaconis. Essa conclusão pode ser estendida aos demais ativos (todos ações). 